\title{
Leseunterricht und seine Orientierungen nach der PISA-Studie. Konzeptionen und Praktiken im Spannungsfeld von alltagsbezogener Kompetenzorientierung und Enkulturation
}

\section{Andrea Bertschi-Kaufmann}

In der Folge von PISA lässt sich eine zunehmende Orientierung an einer Vorstellung von Lesekompetenz beobachten, die Lesen auf messbare Teilkompetenzen herunterbricht. Diese Vorstellung gründet in einem kognitionstheoretischen Modell des Leseprozesses, wobei Lesen als soziale Handlungsfähigkeit konzeptualisiert und auf alltägliche Anwendungssituationen bezogen wird. So einleuchtend diese Vorstellung insbesondere auch für eine vergleichende Leistungsmessung ist, so ist sie doch für den heutigen Leseunterricht problematisch. Zum einen blendet sie die unter der Erwerbsperspektive wichtigen emotionalen und motivationalen Aspekte des Lesens aus, und zum anderen steht sie unvermittelt Bildungstraditionen gegenüber, welche dem literarischen Lesen Bedeutung beimessen. Die Leseforschung gibt zahlreiche Hinweise darauf, dass der Leseunterricht auf der Sekundarstufe I als Zusammenspiel von Lesetraining, Leseförderung und literarischem Lesen gestaltet werden soll, wobei die Schulpraxis auf verlässliche Aussagen zur jeweiligen Wirksamkeit angewiesen ist.

In der Diskussion über eine ausreichende und effektive Sprachförderung hat sich «PISA» weit über die periodischen Aussagen zu den Leseleistungen am Ende der obligatorischen Schulzeit hinaus als Stichwort für ganz verschiedene Probleme und Herausforderungen etabliert: für die unzureichende Ausbildung der Schlüsselkompetenz Lesen (Falter, Pasche \& Hertig, 2007; Rychen \& Salganik, 2003) und sozusagen als Antwort darauf für die Bedeutung eines Gesamtsprachenkonzepts (EDK, 2004), für die Notwendigkeit der sprachlichen Integration von immigrierten Erwachsenen und deren Kindern (EDK, 2004; OECD PISA, 2003), für allfällige schulsystematische Anpassungen nach dem Vorbild anderer Länder (Baumert et al., 2003; Larcher \& Oelkers, 2003), für eine gewünschte Qualitätsentwicklung des Unterrichts (Helmke, 2007, 2009; Oelkers \& Reusser, 2008) und in diesem Zusammenhang für die Bereitstellung hilfreicher Lehrmittel. Unterricht ist also nur eine der Ebenen, auf welcher die Frage nach der Verbesserung und Stabilisierung der Lesekompetenz behandelt wird. Diese ist allerdings deshalb zentral, weil hier ganz verschiedene Grössen zusammenspielen: die bildungsplanerischen Massnahmen (u.a. die Festlegung von 
Sprachlektionen in der Stundentafel), die Lehrpläne, Lehrmaterialien, das Handeln der Lehrerinnen und Lehrer, die Aktivitäten der Schülerinnen und Schüler, letztere beeinflusst von Motivationen und Aspirationen im engen Kontext der unterschiedlichen sozialen Ausgangslagen. Im angelsächsischen und im deutschsprachigen Raum hat man die Unterrichtsqualität im Hinblick auf ihre Wirkung für den Bereich Lesen mit der Evaluation von Förderprogrammen (National Institute of Child Health and Human Development [NICHD], 2000) und mit Studien wie DESI (DESI-Konsortium, 2008) und PIRLS (Mullis, Martin, Kennedy \& Foy, 2007) fokussiert, Brennpunkt ist jeweils der messbare Zuwachs der Leseleistung.

Im Zuge von PISA interessiert «Lesen» im Zusammenhang mit Unterrichtsqualität und Unterrichtspraxis aber auch unter weiteren Gesichtspunkten: es muss danach gefragt werden, welche Bildungskonzeption zum einen den Leistungsvergleichsstudien zugrunde liegt und zum anderen für die Ausrichtung des Unterrichts bestimmend sein soll. Daran anschliessend erklären sich der jeweils wegleitende Lesebegriff bzw. die Modellierung der Lesekompetenz und schliesslich interessiert die für Beobachtung und Einschätzung der Praxis entscheidende Frage, welche Art der Konzeptualisierung von Lesen und Lesekompetenz dort wegleitend ist: für die Lehrerinnen und Lehrer, sichtbar in der Wahl von Unterrichtsmethoden und in der textbezogenen Kommunikation mit den Schülerinnen und Schüler und für diese selber, sichtbar in der Art ihrer Verwertung von unterrichtlichen Angeboten und in Lektüreergebnissen.

Mit Bezug zur Sekundarstufe I in der deutschsprachigen Schweiz geben einzelne Studien Auskunft u.a. über die Wirkungen von Unterrichtsmethoden auf die Lesekompetenz (vgl. Bertschi-Kaufmann \& Schneider, 2006; BertschiKaufmann \& Schneider, 2007), über die Kommunikation in den Erst- Zweitsprachen Deutsch und Französich (Pekarek Doehler, 2009) sowie über die Entwicklung der Lesekompetenz bei Jugendlichen mit ungünstigen Voraussetzungen (Schneider et al., 2009). Im Unterschied zur Romandie, für die systematische Bestandesaufnahmen von Unterrichtspraktiken in den Bereichen Sprache und/oder Lesen vorliegen (vgl. Aeby Daghé \& Dolz, 2007; Schneuwly, Dolz \& Cordeiro, 2005; Soussi, Petrucci, Ducrey \& Nidegger, 2008), ist man für die deutschsprachige Schweiz auf Daten aus einzelnen Forschungsprojekten mit ihren je spezifischen Fragen angewiesen sowie weiter auf Beobachtungen, die im Kontakt mit den Deutschlehrerinnen und -lehrern gewonnen wurden. Einen weiteren Aspekt eröffnet der Blick auf die bildungspolitischen und fachdidaktischen Vorgaben für den Leseunterricht, konkret in den zur Zeit gültigen Lehrplänen (Bättig, 2004), den Standardformulierungen, die im Rahmen des Projekts HarmoS für «Lesen» vorgeschlagen werden (EDK, 2010) sowie in den Lehrmitteln, die mit ihren Unterrichtsvorschlägen meist explizit auf PISA und die in der Studie ausgemachten bzw. ihr zugesprochenen didaktischen Defizite hinweisen und für sich in Anspruch nehmen, effektive Verfahren vorzuschlagen.

Soweit die Bemerkungen zu verschiedenen Bezugspunkten der aktuellen 
Diskussion. Ausgangspunkt der nachfolgenden Überlegungen ist PISA bzw. sind die Orientierungen, welche der internationale Leistungstest für den Bereich Lesen vorgibt (1), wobei dem für PISA relevanten literacy-Konzept anschliessend jenes einer traditionell ausgerichteten Lesebildung gegenübergestellt wird (2). In diesem Spannungsfeld werden die im Rahmen des HarmoS-Projekts neu ausgearbeiteten Kompetenzbeschreibungen und die Standards verortet (3). Die wesentlichen Aufgaben des Leseunterrichts lassen sich aus den genannten Grundlagen ableiten, wobei hier auch die Frage nach der Wirksamkeit der Unterrichtsmethoden gestellt wird (4). Und schliesslich bleibt die wohl anspruchsvollste Aufgabe der Lehrerinnen und Lehrer, Lerndiagnosen zu stellen, Lernentwicklungen im Zusammenhang eines kontinuierlichen Prozesses und ausgerichtet auf ein Lesen als kulturelle Praxis zu verstehen und entsprechend anzuregen (5).

\section{Im Zuge von PISA: Orientierung hin zur messbaren Leseleistung}

Die Ergebnisse in Bezug auf den Stand der Leseleistung in der Schweiz sind bekannt: Er liegt im Durchschnitt aller beteiligten Länder, ist also leidlich und entspricht in keiner Weise den zunehmend hohen Anforderungen, welche die weiterführenden Schulen auf der Sekundarstufe II, die Berufsausbildungen und die Betriebe sowie die betriebliche und die freiwillige Weiterbildung stellen (Sturm, Hollenstein \& Hilbe, 2009). Die fraglichen Anschlussmöglichkeiten jugendlicher Schulabgängerinnen und Schulabgänger werden denn auch als eines der virulenten gesellschaftspolitischen Probleme wahrgenommen (UNESCO, 2009; für die Schweiz Schweizerische Koordinationsstelle für Bildungsforschung [SKBF], 2010, S. 97ff). Die Schlüsselfunktion der Lesekompetenz ist aber über die Laufbahnchancen innerhalb des Bildungssystems und die berufliche Beschäftigung hinaus generell für die soziale Mitgliedschaft wichtig. Allerdings steht die Bearbeitung dieses Problems, die Ausrichtung literaler Bildung auf Partizipation, noch aus: Die Bedeutung des kulturellen Anschlusses ist im Kontext von PISA in Kommentaren zwar hin und wieder erwähnt (z.B. Hurrelmann, 2004), findet aber selten Berücksichtigung in jener Diskussion, die auf schulsystematische Fragen (z.B. Larcher \& Oelkers, 2003) oder auf mehr oder weniger isolierte schulische Massnahmen fokussiert (Kämper-van den Boogaart, 2004). Die Leselern- und Sozialisationsprozesse von Heranwachsenden hin zur Teilhabe an literalen Kulturen sind von schulischen Bedingungen mitgestaltet, das trifft selbstverständlich zu. Sie vollziehen sich allerdings unbestreitbar immer schon in Medienkontexten. Die Entwicklung von diesbezüglichen Praktiken, die insbesondere im anglo-amerikanischen Raum als "partizipatorische Kultur» (Jenkins, 2008) beobachtet und als zukunftweisend gewürdigt werden, nimmt die Institution Schule allerdings nur zum Teil auf 
(Bertschi-Kaufmann \& Wiesner, 2009). Damit ist eines der zentralen Passungsprobleme der Vermittlung von Lesekompetenz angesprochen, jenes zwischen der schulischen, auf einzelne Textformate bezogenen Bildungsnorm und der literalen, medienbezogenen Praxis von Heranwachsenden, von denen die Leistungsschwächeren offensichtlich nicht in der Lage sind, die ausserschulischen Lese- und Medienerfahrungen für den Aufbau schulisch geförderter Lesekompetenz zu nutzen (Bertschi-Kaufmann \& Rosebrock, 2009, S. 13ff.). In engem Zusammenhang damit steht ein weiteres, noch grundlegenderes Passungsproblem, jenes zwischen dem bei der schulischen Beschäftigung mit Texten in aller Regel vorausgesetzten Sprach- und Weltwissen (Grzesik, 2005) und den Spracherfahrungen, auf denen Kinder und Jugendliche tatsächlich aufbauen können. Sprachwissen und Spracherfahrung sind beide sozial gebunden. Der starke Zusammenhang zwischen sozialem Hintergrund und Schulerfolg ist bekannt. In der Schweiz ist er im internationalen Vergleich gar besonders stark (OECD PISA, 2003; Fredriksson, Holzer, McCluskey \& Tabue, 2009) und somit eine verbesserte, sozial integrierende Sprachförderung ein dringliches Desiderat. Dass eine solche nur auf der Grundlage eines umfassenden Konzepts angegangen werden kann und auf schulischer Seite sowohl schulsystematische als auch unterrichtliche Fragen aufgreifen muss, liegt auf der Hand.

Die Bildungspolitik, konkret die Schweizerische Konferenz der kantonalen Erziehungsdirektorinnen und -direktoren (EDK), hat denn auch mit einem strukturierten Massnahmenkatalog reagiert, dem "Aktionsplan PISA 2000" (EDK, 2003), der auf der Grundlage mehrerer Vertiefungsstudien im Anschluss an die Ergebnisse aus PISA 2000 erarbeitet wurde und Bereiche definiert, für welche ein Handlungsbedarf ausgemacht worden war (Rhyn, 2003).

$\mathrm{Zu}$ diesen gehören u.a. die «Sprachförderung für alle», die «Sprachförderung für Kinder und Jugendliche mit ungünstigen Voraussetzungen», die «Schulführung, Förderung der Schulqualität» - Handlungsfelder also, die in der Volksschule situiert werden. Die betreffenden Massnahmen sollten sich nicht auf den Bereich Lesen beschränken, sondern zur Verbesserung der Sprachkompetenz «im umfassenderen Sinn» beitragen (Rhyn, 2003, S. 423). Die Integration von Lesefähigkeit in eine Gesamtsicht der sprachlichen Förderung überzeugt in theoretischer und empirischer Hinsicht: Lesefähigkeit ist eng an sprachliche Kompetenzen wie Wortschatz (Schnotz \& Dutke, 2004), an syntaktisches Wissen und an den Sinn für die Spezifität von Textarten, an ein sog. Genrebewusstsein gebunden (Dolz \& Schneuwly, 1996; Giasson 1990). Aus der Erkenntnis solcher Bezüge von allgemeiner Sprachfähigkeit und der Lesekompetenz lässt sich allerdings noch keine Fördertätigkeit zwingend ableiten. Wenn der «Aktionsplan PISA 2000» - im Rahmen der Aufzählung konkret zu ergreifender Massnahmen - die Verwendung der Standardsprache im Unterricht (EDK, 2003, S. 7) oder die Verbesserung der Diagnosefähigkeit von Lehrpersonen (EDK, 2003, S. 13) vorschlägt, dann spricht dafür eine gewisse Plausibilität; es fehlt aber ein Nachweis dafür, wie stark und unter welchen Vorausset- 
zungen entsprechende Interventionen wirken, es fehlt die Evidenz. Diese aber brauchen die Schulen dringend, wenn sie darauf verpflichtet werden sollen, Schüler und Schülerinnen zu besseren (Lese-)Leistungen zu führen. Bei aller Wahlfreiheit der Unterrichtsmethoden, auf die als positive Kehrseite der Verantwortung für den so genannten Outcome immer wieder hingewiesen wird (z.B. Oelkers \& Reusser, 2008), ist doch entscheidend, ob den Schulen und den Lehrerinnen und Lehrern ein Wissen über deren Effektivität zur Verfügung steht oder ob sie auf mehr oder weniger verlässliche Erfahrungen aus einer als gut eingeschätzten Praxis angewiesen sind. Beim jetzigen Stand der Unterrichtsforschung hingegen ist der Anspruch, sich innerhalb des Ratings von Lesekompetenz im Ländervergleich zu verbessern, weitaus höher als das Wissen in Bezug auf die Wirksamkeit verschiedener Massnahmen der Leseförderung.

Vergegenwärtigen wir uns die Vorgaben, die aus den Schweizer Ergebnissen aus PISA 2000 bezogen auf die sprachlichen Fähigkeiten abgeleitet wurden, so fällt die Orientierung an quantifizierbaren Zielen auf. Dreierlei sollte erreicht werden, nämlich

- die Steigerung der durchschnittlichen Lesekompetenz,

- die Reduktion der Unterscheide in der Lesefähigkeit und

- die Reduktion des Anteils der Jugendlichen, die massive Lernschwierigkeiten haben (Rhyn, 2003, S. 243).

Von den Schweizer Schülerinnen und Schülern wird also erwartet, dass sie sich insgesamt verbessern, wobei Spitzenergebnisse vermehrt und die Leistungen am unteren Ende der Skala ihrerseits verbessert werden sollen.

So verständlich diese skalenbezogenen Zielformulierungen im Kontext der Evaluation von Bildungssystemen im Ländervergleich sind, so problematisch sind sie auch. Es geraten nämlich jene Bereiche der Leseentwicklung in der auf PISA gerichteten Diskussion aus dem Blick, die zwar entscheidend für das Gelingen von Lesenentwicklung sind, deren Beitrag zum Gelingen aber nicht in messbaren Kategorien aufgeht und die deshalb als blinde Flecken untergehen: Dabei handelt es sich zum einen um das Vorwissen, angesammelt im Verlauf von Prozessen der Welt- und Spracherfahrung (Bruner, 1990), und zum anderen um die Lesemotivation der Leserinnen und Leser, die sie auf der Erfahrung mit literalen Praktiken aufbauen und die mit der Leseleistung zwar korrespondieren, aber sich darin nicht einfach abbilden.

In den Kreisen der Bildungsadministration, der Leseforschung und der Lesedidaktik hat PISA denn auch Kontroversen bezüglich der Reichweite, der Gewichtung und der Qualität von Leistungsüberprüfung in Form von Tests und so genannten Checks (Bayer \& Keller, 2009) ausgelöst. In Bezug auf den Kompetenzbereich Lesen besteht insbesondere ein Spannungsfeld zwischen 
einer Orientierung am Outcome messbarer Leseleistung nach Massgabe psychometrischer Methodik (z.B. Beck \& Klieme, 2007) und der Aufmerksamkeit für die Genese von Lesekompetenz mithin für die Verlaufsentwicklungen des Lesenlernens. Die Lesesozialisationsforschung (Groeben \& Hurrelmann, 2004) macht mit einschlägigen Arbeiten deutlich darauf aufmerksam, dass mit der Erwerbsperspektive weitere Facetten der Lesekompetenz in den Blick geraten, wobei neben kognitiven und reflexiven Dimensionen der Lesekompetenz auch die Lesemotivation und die emotionale Beteiligung am Gelesenen eine zentrale Rolle spielen. Diese auszublenden, ist weder theoretisch richtig noch pädagogisch sinnvoll, weil Lernprozesse im kognitiven Bereich bekanntlich nicht isoliert verlaufen, sondern Teil eines Bedingungsgefüges sind. Die Sprach- und Lesedidaktik darf deshalb ihren Bereich - folgt man der überzeugenden Argumentation der Lesesozialisationsforschung - «nicht vorab um zentrale Dimensionen verkürzen»(Hurrelmann, 2009), weil sie es mit einem komplexen Gefüge gesellschaftlicher Bildungsnormen, bildungsökonomischer Interessen, sozialer Einflussgrössen, individueller Ausgangslagen und für das Lesen relevanter individueller Merkmale zu tun hat.

\section{Lesekompetenz: Literacy vs tradierte Lesebildung}

PISA hat die Schule getroffen, und dies nicht einfach nur deshalb, weil ihr mit den periodischen Ergebnissen Defizite vorgehalten wurden - solche waren bereits mit früheren Studien wie IEA (Notter, Meier, Nieuwenboom, Rüesch \& Stoll, 1996) und IALS (Notter, Bonerad \& Stoll, 1999) zutage getreten -, sondern weil die Massstäbe, anhand derer die fehlenden Kompetenzen festgehalten wurden, nicht im Einklang standen mit den Curricula, denen das Schulfach Sprache (in der deutschsprachigen Schweiz: das Fach Deutsch) bislang gefolgt war.

Inzwischen ist reading literacy im Sinne von PISA als Begriff populär geworden und hat starke Wirkung entfaltet. Gemeint ist damit die Fähigkeit «geschriebene Texte zu verstehen, zu nutzen und über sie zu reflektieren, um eigene Ziele zu erreichen, das eigene Wissen und Potenzial weiterzuentwickeln und am gesellschaftlichen Leben teilzunehmen» (Deutsches PISA-Konsortium, 2001, S. 23). In der englischen Literatur zu PISA wird reading literacy als reading to learn konzeptualisiert. Damit wird über die kognitive Fähigkeit hinaus, die in der Praxis oft als «Leseverstehen» bezeichnet wird, Lesen als eine soziale Handlungsfähigkeit noch deutlicher als in der deutschen Version akzentuiert: der im Hinblick auf die eigenen Handlungsziele effektive Umgang mit Texten. Gemeint ist also ein auf alltägliche Anwendungssituationen bezogener Begriff von Lesekompetenz. Dieser orientiert sich, wie die meisten zur Zeit aufmerksam diskutierten lesebezogenen Konzepte, an einem kognitionstheoretischen Modell des Leseprozesses, wie es von Kintsch (1998) vorgeschlagen und anschliessend 
weiter geführt wurde (Christmann \& Groeben, 1999; Grzesik, 2005). Lesen gilt auf diesen Grundlagen als ein kognitiv konstruktiver Vorgang, der die aktive Konstitution von Bedeutungen verlangt.

Anhand dieses kognitionstheoretischen Lesemodells der Lesepsychologie lassen sich die Aktivitäten der Lesenden aufschlüsseln in verschiedene Anforderungen, die im Vorgang des Lesens parallel vollzogen werden: die Wort- und Satzidentifikation, die Bildung von Kohärenz zum einen auf lokaler Ebene durch das Verknüpfen von Satzfolgen und zum anderen die globale Kohärenzherstellung, in deren Verlauf Kohärenzen in reduzierenden Organisationsvorgängen verdichtet und miteinander verknüpft werden, so dass ein «Lesefluss» (Topping, 2006) entsteht. In seinem Verlauf bilden Lesende globale textgestützte Hypothesen, die auf das als wichtig Beurteilte gerichtet sind, eine innere Repräsentation des gerade Gelesenen, ein sogenanntes mentales Modell, in welches neue Textmomente ständig Eingang finden, so dass es korrigiert und differenziert wird.

Soweit eine sehr knappe Darstellung des an der Kognitionspsychologie orientierten Modells, dem PISA folgt und auf dessen Grundlage fünf Arten der Verstehensleistung auf zunehmend anspruchsvolleren Ebenen unterschieden und mit Testaufgaben operationalisiert werden: ein allgemeines Verständnis des Textes entwickeln (1), Informationen ermitteln (2), eine textbezogene Interpretation entwickeln (3), über den Inhalt des Textes reflektieren (4), über die Form des Textes reflektieren (5) (Artelt, Stanat, Schneider, Schiefele \& Lehmann, 2004, S. 143).

Für das Messen von Leseleistung ist das im Prinzip ein adäquates Schema, wobei sich seine Differenziertheit empirisch nicht belegen liess und es zumindest in der Berichterstattung auf ein Dreierraster konzentriert wurde (Baumert et al., 2001, S. 89). Allerdings: Wäre der Vorgang des Lesens damit hinreichend beschrieben, liesse sich kaum erklären, warum Heranwachsende bereit sein sollten, sich seinen komplexen Anforderungen zu stellen. Es braucht Antriebe, um die vielschichtigen Denkakte des Lesens einzuüben, es braucht das Engagement des lesenden Individuums, welches sich von seinen Lektüren ausreichende Gratifikationen verspricht und deshalb bereit ist, auf die Schwierigkeiten einzugehen, die Texte einem schnellen Verstehen oft entgegenhalten. Die Lesemotivation - darunter wird die Fokussierung persönlicher Leseziele ebenso verstanden wie die Erfahrungen des Lesevergnügens - ist deshalb konstitutiv für die Leseentwicklung. Lesemotivation und die emotionale Beteiligung am Gelesenen sind denn auch in der Lesesozialisationsforschung als wesentliche Elemente der Lesekompetenz konzeptualisiert (Hurrelmann, 2002a). Motivationen und Emotionen im Umgang mit Texten können und sollen von der Schule stimuliert werden, auf ihre Bedeutung für die erfolgreichen Lesekarrieren wird auch nachdrücklich hingewiesen (Hurrelmann, 2006; Möller \& Schiefele, 2004). Sie sind aber nicht systematisch anleitbar und es gibt bislang auch nur erste methodologische Versuche, mit welchen motivationale und emotionale 
Leseleistung textbezogen gemessen werden (z.B. Bertschi-Kaufmann \& Schneider, 2006). Insbesondere in diesen Bereichen fallen Test- und Unterrichtstraditionen auseinander. Der Leseunterricht, der meist als wesentlicher Teil des Sprachunterrichts geführt wird, ist in dessen kulturelle Entwicklung eingebunden. Es lohnt sich daher, sich die herkömmliche Vorstellung von schulischem Lesen zu vergegenwärtigen - auch gerade in der Reflexion über PISA und seine Implikationen für die Unterrichtspraxis: Schriftlernen wird in dieser Perspektive traditionell zu einem wesentlichen Teil als ein Lernen im Umgang mit der «schönen» Literatur verstanden; in seinem Zentrum sollen die kulturell und individuell bedeutsamen Texte stehen. So verstanden ist Lesen ein Beitrag zur Persönlichkeitsbildung und der Deutschunterricht folgt mit dieser Zielsetzung einem Bildungsbegriff in idealistischer Tradition, die auf das Humboldtsche Bildungsideal zurückgeht und der Sprache bzw. den ausgewählten Texten eine die Sicht auf die Welt und auf die eigene Person formende Kraft zuspricht. Leseverstehen ist selbstverständlich die Voraussetzung und zugleich ein wichtiger Bestandteil einer sprachlich-literarischen Grundbildung; ein entsprechender Hinweis findet sich auch im Bericht des Deutschen PISA-Konsortiums (Baumert et al., 2001, S. 21). Mit der Ausrichtung auf literarische Qualität und textbezogene Erfahrung folgt der Deutschunterricht deutlich anderen Aufgaben als eine auf definierte skills fokussierte Schulung, welche PISA nahe legt.

\section{Lehrpläne, Kompetenzbeschreibungen und Bildungs- standards: Gewichtungen und Präzisierungen für den Bereich Lesen}

Eine Übersicht über die in den Schweizer Kantonen bzw. Regionen zur Zeit gültigen Lehrpläne (Bättig, 2004) fächert für den Bereich der Rezeption schriftlicher Texte einen Zielkatalog auf, der basale Lesefertigkeiten, alltagsbezogene Leseaktivitäten (z.B. das Lesen von Beschreibungen) und die Begegnung mit literarischen Formen (z.B. das Lesen von Erzählungen) umfasst. Während erstere, die Ausbildung von Lesefertigkeiten, für die Stufen der Primarstufe weit häufiger genannt werden als für die Sekundarstufe I, verhält es sich mit den auf bestimmte Texterfahrungen angelegten Nennungen gerade umgekehrt: Alltagsbezogene und literarische Lektüren sind - folgt man den Lehrplanangaben zentral im Sprachunterricht der Sekundarstufe I. Selbstverständlich beschreiben diese Lehrplanvorgaben erst einmal die gewünschte und noch nicht die realisierte Unterrichtspraxis (Künzli et al., 1999); immerhin dokumentieren Lehrplaninhalte das Ergebnis von Aushandlungsprozessen, in welche die Sichtweisen der Fachdidaktik, der Praktikerinnen und Praktiker und jene der Bildungsadministration eingeflossen sind. 
Die Gesamtsicht auf die bestehenden und also längst ausgehandelten Lehrplanvorgaben ist damit Bestandesaufnahme und zugleich einer der Ausgangspunkte für das auf die Harmonisierung der Schweizer Schule und auf die Qualitätsentwicklung des Unterrichts angelegte Nationale Projekt HarmoS. Die im Rahmen von Konsortien beauftragten Expertinnen und Experten hatten Befunde auf ganz verschiedenen Ebenen zu berücksichtigen: jene aus der Dokumentenanalyse bestehender Bildungsvorgaben; Grundlagen, auf welche der internationale Leistungsvergleich PISA aufbaut und schliesslich jene auf Unterrichtstraditionen und Unterrichtserfahrungen gestützten Einschätzungen davon, was sich als bewährte Praxis etabliert hat und woran die weiteren Entwicklungen anschliessen sollten.

HarmoS bzw. die Kompetenzbeschreibungen, die in seinem Rahmen u.a. für den Bereich erste Schulsprache erarbeitet wurden (Konsortium HarmoS Schulsprache, 2010) und die als Grundlage für die Standardformulierungen dienen, sind also dieser Mehrperspektivität verpflichtet. Was den Bereich Lesen und die oben kurz wiedergegebenen Konzeptionen in kognitivistischer und in erfahrungsbezogener Sicht betrifft, so wird in der Tat beidem Rechnung getragen: Orientiert an einer Grundkonzeption des Sprachhandelns wird Sprachkompetenz zunächst allgemein verstanden als «Fähigkeit, mit Sprache situationsangemessen zu handeln» (Konsortium HarmoS Schulsprache, 2010, S. 2) und Lesekompetenz umfasst sowohl die alltagsfunktionalen textbezogenen Handlungen als auch jene des involvierten Lesens von poetisch gestalteten Texten. Lesen gilt in dieser Konzeption mithin als kognitiver und als emotionaler Akt.

Das Konsortium, welches das Lesekompetenzmodell als Teil des Sprachkompetenzmodells, wie es für die künftige fachdidaktische Entwicklungsarbeit in der Schweiz wegleitend sein soll, verantwortet, hat dafür Formulierungen gesucht, die auf die Praxis des Leseunterrichts zielen (Konsortium HarmoS Schulsprache, 2010, S. 5):

Lesen meint die Tätigkeit, Texte unterschiedlicher Textsorten (literarische Texte und Sachtexte, kontinuierliche und diskontinuierliche Texte etc.) sinngemäss auf verschiedenen Ebenen verstehen zu können (Fertigkeiten), Texte als Ausdruck einer Kultur wahrzunehmen und als ästhetisches Produkt geniessen zu können (Ästhetik/Kultur) sowie das Gelesene in der eigenen Vorstellung ausgestalten (Imagination), über das Lesen nachdenken (Reflexion) und sich mit anderen darüber austauschen zu können (Anschlusskommunikation).

Damit setzt HarmoS einen bewusst weit konzipierten Lesebegriff. Kognitive Verstehensleistungen, die Fähigkeit zum literarästhetischen Genuss, die Vorstellungsbildung, Reflexionsfähigkeit und die textbezogene Kommunikation stehen nicht nur für verschiedene Dimensionen einer gegenüber PISA erweiterten Kompetenzdefinition, sondern auch für eine Konzeptualisierung, deren Komponenten nur zum Teil mit quantitativen Verfahren bemessen werden können. 
Standards hingegen, die ihrer Definition nach als massgebliche Normen anzulegen sind, lassen sich selbstredend nur für jene Bereiche der Lesekompetenz festlegen, in denen sichtbare Leistungen gemessen und die Masse als Indikatoren von jeweiligen Entwicklungsständen gelten können. Dies trifft für die kognitiven Lesefähigkeiten durchaus zu, aber nur mit Einschränkungen für die emotionalen und die motivationalen Lesefähigkeiten.

Die Standardformulierungen, die für den Bereich Lesen und jeweils bezogen auf die verschiedenen Phasen der Volksschule vorgeschlagen werden, sind deshalb zwangsläufig enger bzw. beschränkt auf die Ausbildung bzw. die Überprüfung weitgehend kognitiver Leseleistung. Augenfällig ist dies in den Standardformulierungen, welche die EDK auf der Grundlage der erarbeiteten Kompetenzbeschreibungen vorschlägt, zum Beispiel beim Standard «Lesen für das vierte Schuljahr» (EDK, 2010, S. 11), der sich auf die basalen kognitiven Lesefähigkeiten konzentriert. Sichtbar bleibt die Fokussierung auf kognitive Leistungen aber auch in der Standardformulierung, welche für den Bereich Lesen im 11. Schuljahr vorliegt:

Basisstandard Lesen für die 11. Klasse (EDK, 2010, S. 59):

Die Schülerinnen und Schüler können Texte mit vertrautem Thema und schulnahem Wortschatz verstehen, wenn sie mithilfe von passenden Fragen zum Textverstehen angeleitet werden. Sie können die Informationen im Text finden - vorausgesetzt, sie werden zu einem Teil ausdrücklich im Text genannt. Sie können Beziehungen zwischen Abschnitten eines Textes aufgrund eines globalen Textver-stehens herstellen. Sie können dabei die Organisation eines strukturierten Textes nachvollziehen, das Genre und die Textfunktion erkennen. Sie können den Zusammenhang von Text, Bild, Grafik und Tabelle erkennen. Sie können dadurch einen strukturierten Text und sein Thema im Wesentlichen als Ganzes verstehen und mit ihrer Lebenswelt in Verbindung bringen.

Die vergleichsweise enge Formulierung mag erstaunen, dies insbesondere vor dem Hintergrund des oben diskutierten, erweiterten Lesebegriffs und dem dringenden Desiderat, im Hinblick auf die Förderung der Lesekompetenz entscheidende Faktoren wie die Lesemotivation und die ästhetische Erfahrung miteinzubeziehen. Auf der anderen Seite ist die Präzisierung im Hinblick auf operationalisierbare Lesefähigkeiten gerade schlüssig, weil Operationalisierung als Voraussetzung für Standardisierung gilt. Die Standardvorschläge für Lesen sind - wie alle anderen - eingepasst in das Grundkonzept von so genannten Basisstandards, für die man sich von Seiten der EDK sinnvollerweise entschieden hat (HarmoS-Konkordat, EDK, 2007). Als Standard wird das für den Bildungserfolg jeweils Notwendige vorgegeben - und die kognitiven Leseleistungen gehören dazu - nicht aber vollständig alles Hinreichende bzw. die Kompetenz mit allen Aspekten. Für die Praxis zeigen Basisstandards also Niveaus in Kompetenzbereichen an, die als Voraussetzung für weitere Lernschritte mithin als anschlussfähig gelten und für die auf empirischer Basis überhaupt Niveaus beschreibbar sind und festgelegt werden können. Die 
weiteren Anforderungen im Bereich Lesen und insbesondere die Anforderungen an einen effektiven, die Lesetätigkeiten und die Lesefähigkeiten stärkenden Unterricht sind damit nicht definiert. Sie ergeben sich zum einen auf der Grundlage der Ergebnisse der Leseforschung und zum anderen aus den Erfahrungen ausgewiesener Praxis.

\section{Der "Guter Leseunterricht" und seine Aufgaben: Auf der Suche nach Evidenz}

Kombiniert man die verschiedenen Perspektiven der auf den Nachweis kognitiver Leseleistung fokussierten Diskussion über Unterrichtsqualität einerseits mit einer Orientierung an den heranwachsenden Leserinnen und Lesern und ihren text- und medienbezogenen Interessen andererseits und schliesslich mit literarästhetischen Bildungszielen, so sind es vor allem drei Aufgaben, die dem Sprachunterricht, speziell dem Lesunterricht übertragen werden:

Das Lesetraining, mit welchem Teilprozesse des Lesens eingeübt und automatisiert werden und für welche nach dem Vorbild angloamerikanischer Trainingsprogramme Lehrmittel mit systematisch aufgebauten Übungen mittlerweile auch im deutschsprachigen Raum und auch für die oberen Klassen der Primarschule sowie für die Sekundarstufe I entwickelt wurden (z.B. BertschiKaufmann, Hagendorf, Kruse, Rank, Riss \& Sommer, 2007; Druschky et al., 2004; Trenk-Hinterberger, Nix, Rieckmann, Rosebrock \& Gold, 2008). Letzteres ist für die deutschsprachige Schweiz insofern neu, als man lange Zeit davon ausgegangen war, dass basale Lesefähigkeiten bei den Schülerinnen und Schülern spätestens am Ende ihrer Primarschulzeit gesichert und Leseübungen anschliessend nicht mehr angezeigt seien. Die Ergebnisse der internationalen Leistungsvergleiche haben dieser Annahme deutlich widersprochen, und viele Lehrmittelverlage haben sich mit einer Fülle von neuen Übungsmaterialien in der Folge insbesondere auf PISA berufen. Die Materialien enthalten mehrheitlich Übungen zum Erwerb von mechanisierbaren Lesefertigkeiten und von planbaren Lesestrategien. In der angloamerikanischen Leseforschung hingegen hat sich ein Forschungszweig etabliert, der unter dem Begriff der «Fluency» jenen Leseprozessen nachspürt, welche über die Dechiffrierfähigkeit hinaus zu hierarchiehöheren Leistungen führt (Rosebrock \& Nix, 2006; Wolf \& Katzir-Cohen, 2001). Unter «Fluency» wird ein auf der Wort- und Satzebene automatisierter, müheloser und ausdrucksstarker Lesefluss verstanden. Lesegeläufigkeit gilt als wesentliche Voraussetzung für das Textverstehen und sie gilt als sukzessive trainierbar.

Die allgemeine Leseförderung, mit welcher - in einem weiten Verständnis - die Stimulierung jeglicher Leseaktivität gemeint ist und deren Ziel in erster Linie das Wecken von Leseinteressen und die Stabilisierung des Leseverhaltens ist. Mit Konzepten der Leseanimation, der Verstärkung von Leseroutinen bzw. der Habitualisierung von Lesen im Rahmen des Unterrichtsalltags werden Leseum- 
gebungen so eingerichtet, dass den heranwachsenden Leserinnen und Lesern Raum für individuelle Entscheide bleibt, sie dabei aber auch auf Hinführungen zu ihren Lektüren zählen können (Bertschi-Kaufmann, 2006). Entsprechende Förderkonzepte rücken motivationale Aspekte in den Vordergrund. Sie setzen in der Regel aber nicht nur auf die zu weckende «Leselust», sondern auch auf die wechselseitigen Animationen und Verständigungen über Leseerfahrungen und Leseerkenntisse im Rahmen eines textbezogenen Austauschs, der Anschlusskommunikation, die von der Lesesozialisationsforschung (Hurrelmann, 2002b) als besonders wirksam für den Aufbau von Leseentwicklungen eingestuft wird. Mit dem Ziel der Leseförderung richten Schulen und Bibliotheken Leseecken mit vielerlei Leseangeboten ein, wobei elektronische Medien mittlerweile zum selbstverständlichen Inventar gehören, weil ihr Anregungspotenzial für das Lesen, auch für das Lesen gedruckter Texte, ausgewiesen ist (zum Beispiel Bertschi-Kaufmann, Kassis \& Sieber, 2004).

Das literarische Lernen, welches sich im Umgang mit poetisch gestalteten Texten vollzieht und je nachdem andere Voraussetzungen hat als die allgemeine Lesekompetenz. Während Lesefähigkeit nämlich zeichengebunden ist, gilt dies für die literarische Kompetenz nicht zwingend. Die Rezeption des gesprochenen Kinderverses, der Theaterbesuch, das Hören und Verstehen von Raptexten u.a. sind Elemente des literarischen Lernens; insofern ist dieses zunächst weniger voraussetzungsreich als jegliches rein schriftbezogene Lernen. Der Literaturunterricht, und insbesondere jener der Sekundarstufen I und des Gymnasiums rückt allerdings die anspruchsvolle literarische Lektüre ins Zentrum, und dies mit dem Lernziel, literarische Texte angemessen bzw. «formbewusst» (Eggert, 2002) und traditionsbewusst (Abraham \& Kepser, 2009) zu rezipieren. Texte sollen in ihrer medialen Gestaltung und in ihrem kulturellen Kontext verstanden werden. Während Konzepte der literarischen Erfahrung auf ein unmittelbares Spracherleben im Umgang mit dem Kunstvollen setzen (Härle \& Rank, 2008), meint man mit literarischem Lernen in der Regel eine mehr oder weniger starke Orientierung an Texten, die unter poetologischen und/oder normativen Aspekten ausgewählten wurden, also eine Orientierung am literarischen Kanon. Anders als in Konzepten der Leseförderung gelten hier mehr oder weniger explizite Regeln für die Aufschlüsselung der Texte. Die Rezeptionsprozesse der heranwachsenden Leserinnen und Leser sind in jene Formen des Verstehens und Deutens eingebunden, die kulturell überliefert und von kanonpflegenden Instanzen wie der Schule als Wissensbestand verlangt werden (Rosebrock, 2006, S. 58). Der Erwerb poetischer Kenntnisse ist allerdings nicht Selbstzweck, sondern gilt als Voraussetzung einer vertieften Erfahrung jener Welt- und Sprachvermittlung, die literarische Texte bieten (Paefgen, 2006, S. 158). In diesem Sinne wird der Kinder- und Jugendliteratur als Fundus poetischer Texte mit vergleichsweise leicht zugänglichen Gestaltungen als Übergangsliteratur eine grosse - animierende und didaktische - Bedeutung zugemessen. 
Wenn Lehrerinnen und Lehrer eine Gewichtung der hier kurz skizzierten leseund literaturdidaktischen Aufgaben vornehmen, so entscheiden sie in aller Regel nach Plausibilitäten, die sich aus den Traditionen des Schultyps ergeben (der Anteil literarischen Lernens wird in der Sekundarstufe I mit geringem Anspruch bzw. der Sek C kleiner sein als in progymnasial konzipierten Typen) und von Lehrmitteln oder der eigenen Erfahrung gestützt sind.

Im Nachklang von PISA ist die Frage nach dem nachweislichen Erfolg von Unterrichtskonzepten und -verfahren aber virulent. Die Antworten fallen allerdings aus verschiedenen Gründen spärlich aus, denn zum einen sind die genannten Didaktiken in ganz unterschiedlichem Masse geeignet für eine Überprüfung ihrer Produktivität (jene PISA-Aufgaben, welche sich auf literarische Texte beziehen, sind diesbezüglich auch nicht wegweisend, weil sie wohl inhaltliche und formale Merkmale der Texte gerichtet sind, nicht aber nach dem spezifisch literarischen Verstehen als ein Deuten und Einordnen in die bereits angesammelte literarische Erfahrung fragen). Zum anderen macht die Leseforschung bislang nur wenige eindeutige Aussagen bezüglich der Wirkung von Unterrichtsmethoden im Bereich Lesen. Immerhin verweisen Studien aus ganz unterschiedlichen Kontexten darauf, dass eine Kombination von Unterrichtsverfahren angezeigt ist und dass Interventionen zur Schulung von kognitiven Verstehensprozessen wirksam unterstützt werden durch solche, welche eine freie, aber extensive Lesepraxis anregen. Entsprechende Schlüsse werden zum einen in jenen Kommentaren gezogen, welche sich auf die Metaanalysen zu den amerikanischen Lesetrainingsprogrammen im Rahmen des «National Reading Panels» (NICHD, 2000) beziehen. So deutet Allington (2009) dessen Ergebnisse dahingehend, dass insbesondere schwache Leserinnen und Leser mit Sequenzen eines offen gehaltenen, interessegeleiteten Lesens besser gefördert werden als mit einem ausschliesslich angeordneten Lesetraining. Mit Bezug auf die Lesemotivationsforschung (z.B. Wigfield \& Guthrie, 1997) kommt man in den auf die verschiedenen Interventionsstudien im amerikanischen Raum gezogenen Schlussfolgerungen also auf sehr ähnliche Befunde wie deutsche Forschungsarbeiten, z.B. jene von Möller und Schiefele (2004), Retelsdorf und Möller (2008), deren Ergebnisse sich so zusammenfassen lassen: Lesemenge und intrinsische Lesemotivation hängen eng zusammen, beide unterliegen eher ausserschulischen Faktoren, weil freizeitliches Lesen mehr Selbstbestimmung zulässt, Eine erhöhte Lesemenge wiederum bewirkt ein gesteigertes Leseverständnis.

Für den Leseunterricht ist also eine Aktivierung der Lesetätigkeit, und diese möglichst mit Wirkung auf das freiwillige Lesen ausserhalb der Schule, deutlich angezeigt. Denn Lesenlernen gelingt nie ausschliesslich mit dem Nachvollzug angeleiteter Schritte, sondern ist Ergebnis eines umfassenden Enkulturationsprozesses. Aus der von uns ausgetragenen Studie «Lese- und Schreibkompetenzen fördern» (Bertschi-Kaufmann \& Schneider, 2006, 2007) wissen wir zudem, dass sich Aktivierungen des freien Lesens auch hinsichtlich kognitiver Leseentwicklungen bewähren bzw. dass ein Lesetraining auf der Sekundarstufe I 
(untersucht für den Typus C) mehr und bessere Wirkung zeigt, wenn es mit Sequenzen des freien, interessegeleiteten Lesens kombiniert wird und wenn Lehrerinnen und Lehrer auf die Lernschritte und Förderbedürfnisse ihrer Schülerinnen und Schüler aufmerksam sind.

\section{Diagnosefähigkeit und Lernerfolg: Vom Zusammen- spiel der Kompetenzen von Lehrenden und Lernenden im Leseunterricht}

Diagnostische Kompetenz von Lehrerinnen und Lehrern wird als eine Schlüsselkompetenz in Lehr- und Lernkontexten angesehen; PISA hat diese Beurteilung insofern noch verstärkt, als man auf die Kluft zwischen der Einschätzung von Leseleistungen durch die Lehrerinnen und Lehrer und den in Testergebnissen zutage tretenden Defiziten aufmerksam wurde (Kultusministerkonferenz, 2001). Unter «diagnostischer Kompetenz» wird allgemein die Kompetenz von Lehrerinnen und Lehrern verstanden, Merkmale ihrer Schülerinnen und Schüler angemessen zu beurteilen, Lern- und Aufgabenanforderungen adäquat einzuschätzen. Diagnosefähigkeit der Lehrenden ist die Voraussetzung dafür, dass Förderung bei den tatsächlich gegebenen Voraussetzungen der Lernenden ansetzt und von diesen deshalb verwertet werden kann; sie gilt in diesem Sinne als Angelpunkt der Qualitätsentwicklung von Unterricht. Dem Desiderat verlässlicher diagnostischer Urteile steht allerdings ein wissenschaftlicher Forschungsstand gegenüber, der hinsichtlich der Entstehung, der Förderbarkeit und der Auswirkungen von Diagnosekompetenz bei den Lehrerinnen und Lehrern noch unbefriedigend und für die Praxis wenig hilfreich ist (Artelt $\&$ Gräsel, 2009).

Testaufgaben in Formaten, wie sie für gross angelegte Leistungsstudien angezeigt sind, eignen sich gerade nicht dazu, die für die Entwicklung entscheidenden Aspekte der Lesekompetenz derart zu erfassen, dass daraus direkte Hinweise für eine probate Förderung abzuleiten wären. Die Rückmeldung, die Lehrerinnen und Lehrer mit Testergebnissen erhalten, sind erst einmal nur Hinweise darauf, ob und in welchem Ausmass Schülerinnen und Schüler die gegebenen Aufgaben zum gegebenen Zeitpunkt bewältigt haben (Husfeldt \& Lindauer, 2009). Die Leistungsmöglichkeiten der Lernenden, ihre Dispositionen zum weiteren Leseerwerb und die Ursachen allfälliger Stagnationen sind mit der Testaufgabe nicht zu ermitteln.

Die Sache ist komplexer: Eine geringe Lesekompetenz (in der Definition von PISA) ist unter Umständen Ausdruck allgemeiner Lernschwierigkeiten, ein solcher Zusammenhang ist aber nicht zwingend. Schwierigkeiten, die jemand beim Lesen hat, können unterschiedlicher Natur sein; sie können in fehlendem Vorwissen hinsichtlich des jeweiligen Inhalts oder der spezifischen Textkonventionen und den darauf auszurichtenden Lesestrategien liegen; sie können aber 
auch basale Fertigkeiten im Umgang mit Prinzipien des Schriftsystems und das Konstruieren von Textbedeutungen betreffen. Ergebnisse schlechter Leseleistung verweisen also noch nicht auf die spezifischen Schwachstellen. Um diese zu erkennen, sind Analyseinstrumente für die Förderpraxis erforderlich, wobei einerseits die Voraussetzungen der Schülerinnen und Schüler eingeschätzt werden müssen und andererseits die Anforderungen der jeweiligen Aufgaben, welche ihnen gestellt werden. Im Falle von Leseaufgaben betrifft eine solche Einschätzung zweierlei. Zum einen ist sie auf die Texte bezogen, welche zum Verstehen aufgegeben sind. Diese sind aufgrund ihrer Struktur, der Explizitheit der Leserführung, ihres Wortgebrauchs und ihrer lebensweltlichen Bezüge mehr oder weniger einfach oder schwierig. Und zum anderen sind auch die Aufgaben einzuschätzen, die bezogen auf die Texte gestellt werden: Je nachdem, ob sie Lesewege strukturieren helfen oder direkt nach Endergebnissen von Verstehensprozessen fragen und je nach der Anzahl und der Auswahl von Textsignalen, welche für richtige Antworten berücksichtigt werden müssen, sind sie ihrerseits mehr oder weniger anspruchsvoll.

Während Lehrerinnen und Lehrer der Sekundarstufe I aufgrund ihrer Ausbildung tendenziell auf die Unterscheidung und Einordnung von Textarten gut vorbereitet sind (Soussi et al., 2008), sind die Voraussetzungen zum Erkennen individueller Leseschwierigkeiten nicht unbedingt gegeben. Lange Zeit herrschte nämlich sowohl in der Lehrerinnen- und Lehrerbildung als auch in der Praxis die irrtümliche Meinung, Lesefähigkeiten seien bereits in der Primarschule hinreichend ausgebildet worden und könnten also auf den weiterführenden Schulstufen als selbstverständlich vorausgesetzt werden.

Ein grosses Problem des Leseunterrichts auf der Sekundarstufe I und insbesondere auf der Sekundarstufe C mag darauf zurückzuführen sein; es besteht gerade darin, dass sich Lehrerinnen und Lehrer mit der Anpassung von textseitigen Anforderungen und den Leistungsmöglichkeiten der heranwachsenden Leserinnen und Leser schwer tun bzw. dass der Leseunterricht an den Interessen und den Aufnahmemöglichkeiten der Schülerinnen und Schüler vorbeizielt (Pieper, Rosebrock, Volz \& Wirthwein, 2004). Hier wäre von anderen Ländern und insbesondere von Neuseeland zu lernen, wo Unterrichtsformate und Lehrmittel für eine systematisch angelegte Individualisierung zur Verfügung stehen. Ein Teil des Unterrichts findet in homogenen Lerngruppen statt, wobei Schülerinnen und Schüler aufgrund ihrer jeweiligen Leistungen eingeteilt werden und im Schwierigkeitsgrad angepasste Unterrichtsmaterialien erhalten. Massgeblich für die Zuordnung in die Gruppen sind nicht ausschliesslich Testergebnisse; es werden die Berichte der Lehrerinnen und Lehrer bzw. deren Beobachtungen in den ersten Schulwochen einbezogen. Aufgrund der weiteren Beobachtungen im Verlauf des Schuljahres sind auch immer Umteilungen möglich. 
In Deutschschweizer Schulen hat sich zudem eine Praxis der kontinuierlichen Beobachtung mit Hilfe zweier Instrumente bewährt: dem Beobachtungsraster, mit dessen Hilfe einzelne Teilfähigkeiten des Lesens unterschieden und in den Blick genommen werden können (Bertschi-Kaufmann et al., 2007), und dem Lesetagebuch, welches die Schülerinnen und Schüler begleitend zu den freien Lektüren führen und persönlich gestalten (Bertschi-Kaufmann, 2007). Hier reproduzieren sie ihre Leseerfahrungen in eigenen Texten, verbinden also Lesen mit Schreiben, was den Vorteil hat, dass die beiden literalen Tätigkeiten wechselseitig angeregt und ihrer Verbindung beobachtet werden können.

Ein weiteres Problem besteht in der Tendenz, den Sprach- und Leseerwerb als mehr oder weniger isolierte schulische Aufgabe, statt in seinen lernbiographischen Zusammenhängen zu sehen. Diese auszuleuchten, kann nicht Aufgabe der einzelnen Lehrerinnen und Lehrer sein. Hingegen sollte deren Unterricht Lesepraktiken so anregen, dass diese innerhalb und ausserhalb der Schule weitergeführt und dass Anregungen in beiden Richtungen - vom schulischen Lesen hin zur freizeitlichen Lektüre und umgekehrt - genutzt werden. Denn Leseentwicklungen sind Ausdifferenzierungen der Lesefähigkeiten unter den jeweiligen Bedingungen lebenslanger Literalisierungsprozesse (Holle, 2004, S. 13) und der Leseunterricht leistet günstigerweise einen gewichtigen Beitrag mit anschlussfähigen Teilen im Gefüge sprachlicher und literaler Enkulturation.

\section{Literaturverzeichnis}

Abraham, U. \& Kepser, M. (2009). Literaturdidaktik Deutsch. Eine Einführung (3. Auflage). Berlin: Erich Schmidt.

Allington, R. L. (2009). If they don't read much... 30 years later. In E. Hiebert (Ed.), Reading more, reading better (p. 30-54). New York: The Gilford Press.

Aeby Daghé, S. \& Dolz, J. (2007). Lire pour écrire: Stratégies d'enseignement de la lecture lors de l'écriture de divers genres de textes. Langage et pratiques, 40, 44-52.

Artelt, C., Stanat, P., Schneider, W., Schiefele, U. \& Lehmann, R. (2004). Die PISA-Studie zur Lesekompetenz: Überblick und weiterführende Analysen. In U. Schiefele, C. Artelt, W. Schneider \& P. Stanat (Hrsg.), Struktur, Entwicklung und Förderung von Lesekompetenz. Vertiefende Analysen im Rahmen von PISA 2000 (S. 139 -168). Wiesbaden: VS Verlag für Sozialwissenschaften.

Artelt, C. \& Gräsel, C. (2009). Diagnostische Kompetenz von Lehrkräften. Zeitschrift für Pädagogische Psychologie, 23 (3-4), 157-160.

Bättig, B. (2004). HarmoS. Lehrplanvergleich - Erstprache. Bern: EDK.

Baumert, J., Klieme, E., Neubrand, M., Prenzel, M., Schiefele, U., Schneider, P., Stanat, P., Tillmann, K.-J., Weiss, M. (Hrsg.). (2001). PISA 2000: Basiskompetenzen von Schülerinnen und Schülern im internationalen Vergleich. Opladen: Leske + Budrich.

Baumert, J., Artelt, C., Klieme, E., Neubrand, M., Prenzel, M., Schiefele, U., Schneider, W., Tillmann, K.-J., \& Weiss, M. (Hrsg.). (2003). PISA 2000. Ein differenzierter Blick auf die Länder der Bundesrepublik Deutschland. Opladen: Leske + Budrich.

Bayer, N. \& Keller, F. (2009). Check 5. 2008. Schlussbericht zuhanden des Departements Bildung, Kultur und Sport des Kantons Aargau. Zürich: Institut für Bildungsevaluation.

Beck, B. \& Klieme, E. (Hrsg.). (2007). Sprachliche Kompetenzen. Konzepte und Messung. DESI-Studie (Deutsch Englisch Schülerleistungen International). Weinheim: Beltz. 
Bertschi-Kaufmann, A., Kassis, W. \& Sieber, P. (Hrsg.). (2004). Mediennutzung und Schriftlernen. Analysen und Ergebnisse zur literalen und medialen Sozialisation. Weinheim: Juventa.

Bertschi-Kaufmann, A. (2006). Das Lesen anregen, fördern, begleiten. Velber: Kallmeyer bei Friedrich.

Bertschi-Kaufmann, A. \& Schneider, H. (2006). Entwicklung von Lesefähigkeit: Massnahmen - Messungen - Effekte. Ergebnisse und Konsequenzen aus dem Forschungsprojekt «Lese- und Schreibkompetenzen fördern». Schweizerische Zeitschrift für Bildungswissenschaften, 28 (3), 393-421.

Bertschi-Kaufmann, A., Hagendorf, P., Kruse, G., Rank, K., Riss, M. \& Sommer, T. (2007). Lesen. Das Training. Ausgaben für das 4.-6. und das 7.-9. Schuljahr. Seelze-Velber: Lernbuch Verlag bei Friedrich.

Bertschi-Kaufmann, A. \& Schneider, H. (2007). Freies Lesen oder Lesetraining? Zu den Wirkungen zweier Unterrichtsmethoden auf die Lesekompetenz und -motivation. Ergebnisse der Schweizer Studie «Lese- und Schreibkompetenzen fördern». JuLit, 33 (4), 53-59.

Bertschi-Kaufmann, A. \& Rosebrock, C. (2009). Literalität: Bildungsaufgabe und Forschungsfeld. In Bertschi-Kaufmann, A. \& Rosebrock, C. (Hrsg.), Literalität. Bildungsaufgabe und Forschungsfeld (S. 7-17). Weinheim und München: Juventa

Bertschi-Kaufmann, A. \& Wiesner, E. (2009). Lesealltag und Leseglück in den Selbstaussagen von Jugendlichen. In A. Bertschi-Kaufmann \& C. Rosebrock (Hrsg.), Literalität. Forschungsfeld und Bildungsaufgabe (S. 217-232). Weinheim: Juventa.

Bruner, J. (1990). Acts of meaning. Cambridge, MA: Harvard University Press.

Christmann, U. \& Groeben, N. (1999). Psychologie des Lesens. In B. Franzman, K. Hasemann, D. Löffler \& E. Schön (Hrsg.), Handbuch Lesen (S. 145-223). München: Saur.

DESI-Konsortium (Hrsg.). (2008). Unterricht und Kompetenzerwerb in Deutsch und Englisch. Ergebnisse der DESI-Studie. Weinheim: Beltz Pädagogik

Dolz, J. \& Schneuwly, B. (1996). Genres et progression en expression orale et écrite. Eléments de réflexions à propos d'une expérience romande. Enjeux, 37/38, 49-75.

EDK. (2003). Aktionsplan «PISA 2000» - Folgemassnahmen. Zugriff am 01.03.2010 unter http://edudoc.ch/record/33241/files/pisa2000_aktplan_d.pdf

EDK. (2004). Sprachenunterricht in der obligatorischen Schule: Strategie der EDK und Arbeitsplan für die gesamtschweizerische Koordination (Stand 25.03.2004). Zugriff am 01.03.2010 unter http://edudoc.ch/record/30008/files/Sprachen_d.pdf

EDK. (2007). Interkantonale Vereinbarung über die Harmonisierung der obligatorischen Schule (HarmoS) (Stand 14. Juni 2007). Zugriff am 01.03.2010 unter http://edudoc.ch/record/24711/files/HarmoS_d.pdf?In=deversion=1

EDK. (2010). Basisstandards für die Schulsprache. Unterlagen für den Anhörungsprozess (Stand 25.01.2010). Zugriff am 01.03.2010 unter http://edudoc.ch/record/36475/files/Standards_L1_d.pdf

Eggert, H. (2002). Literarische Bildung und ihre Anforderungen an die Lesekompetenz. In N. Groeben \& B. Hurrelmann (Hrsg.), Lesekompetenz. Bedingungen, Dimensionen, Funktionen (S. 186-194). Weinheim: Juventa.

Falter, J.-M., Pasche, C. \& Hertig, P. (2007). Compétences, formation et marché du travail en Suisse. Une exploitation des résultats de l'enquête internationale sur les compétences des adultes $(A L L)$. Neuchâtel: Office fédéral de la statistique.

Fredriksson, U., Holzer, T., McCluskey, H. \& Tabue, K. (2009). Strength and Weaknesses in the Swedish and Swiss Education Systems; A comparative analysis based on PISA data. European Educational Research Journal, 1, 54-68.

Giasson, J. (1990). La compréhension en lecture. Boucherville: Gaëtan Morin Editeur.

Groeben, N. \& Hurrelmann, B. (Hrsg.). (2004). Lesesozialisation in der Mediengesellschaft. Ein Forschungsüberblick. Weinheim: Juventa. 
Grzesik, J. (2005). Texte verstehen lernen. Neurobiologie und Psychologie der Entwicklung von Lesekompetenz durch den Erwerb von textverstehenden Operationen. Münster: Waxmann.

Härle, G. \& Rank, B. (Hrsg.). (2008). "Sich bilden ist nichts anders, als frei werden.» Sprachliche und literarische Bildung als Herausforderung für den Deutschunterricht. Hohengehren: Schneider.

Helmke, A. (2007). Unterrichtsqualität: Erfassen, bewerten, verbessern. 6. Aufl. Seelze: Kallmeyer.

Helmke, A. (2009). Unterrichtsqualität und Lehrerprofessionalität. Diagnose, Evaluation und Verbesserung des Unterrichts. Seelze: Klett-Kallmeyer.

Holle, K. (2004). Prävention von Leseschwierigkeiten oder wieso das Lesen lernen nicht nur ein grundschuldidaktisches Problem ist. In M. Kämper-van den Boogaart (Hrsg.), Deutschunterricht nach der PISA-Studie (S. 13-35). Frankfurt a. M.: Peter Lang.

Hurrelmann, B. (2002a). Leseleistung - Lesekompetenz. Praxis Deutsch, 176, 6-18.

Hurrelmann, B. (2002b). Prototypische Merkmale der Lesekompetenz. In N. Groben \& B. Hurrelmann (Hrsg.), Lesekompetenz. Bedingungen, Dimensionen, Funktionen (S. 275-286). Weinheim: Juventa.

Hurrelmann. B. (2004). Sozialisation der Lesekompetenz. In U. Schiefele, C. Artelt, W. Schneider \& P. Stanat (Hrsg.), Struktur, Entwicklung und Förderung von Lesekompetenz. Vertiefende Analysen im Rahmen von PISA 2000 (S. 37-60). Wiesbaden: VS Verlag für Sozialwissenschaften.

Hurrelmann, B. (2006). Ein erweitertes Konzept von Lesekompetenz und Konsequenzen für die Leseförderung. In G. Auernheimer (Hrsg.), Schieflagen im Bildungssystem. Die Benachteiligung der Migrantenkinder (2. Auflage) (S. 161-176). Wiesbaden: Verlag für Sozialwissenschaften.

Hurrelmann, B. (2009). Literalität und Bildung. In A. Bertschi-Kaufmann \& C. Rosebrock (Hrsg.), Literalität. Bildungsaufgabe und Forschungsfeld (S. 21-42). Weinheim: Juventa.

Husfeldt, V. \& Lindauer, T. (2009). Kompetenzen beschreiben und messen. Eine Problematisierung selbstverständlicher Begriffe. In A. Bertschi-Kaufmann \& C. Rosebrock (Hrsg.), Literalität. Bildungsaufgabe und Forschungsfeld (S. 137-150). Weinheim: Juventa.

Jenkins, H., Purushotma, R., Clinton, K., Weigel, M., \& Robison, A. J. (2008). Confronting the challenges of participatory culture: media education for the 21st Century. Massachusetts Institute of Technology: MacArthur Foundation.

Kämper-van den Boogaart, M. (Hrsg.). (2004). Deutschunterricht nach der PISA-Studie. Frankfurt a.M.: Peter Lang.

Kintsch, W. (1998). Comprehension: A paradigm for cognition.Cambridge University Press.

Konsortium HarmoS Schulsprache. (2010). Schulsprache. Wissenschaftlicher Kurzbericht und Kompetenzmodell. Provisorische Fassung (vor Verabschiedung der Standards) (Stand 17.01.2010). Zugriff am 01.03.2010 unter http://www.edudoc.ch/static/web/arbeiten/harmos/L1_wissB_25_1_10_d.pdf

Künzli, R., Bähr, K., Fries, A., Ghisla, G., Rosenmund, M. \& Seliner-Müller, G. (1999). Lehrplanarbeit. Über den Nutzen von Lehrplänen für die Schule und ihre Entwicklung. Chur: Rüegger.

Larcher, S. \& Oelkers, J. (2003). Bildungsmonitoring Schweiz. Die besten Ausbildungssysteme. Thematischer Bericht der Erhebung PISA 2000. Neuchâtel: BfS/EDK.

Möller, J. \& Schiefele, U. (2004). Motivationale Grundlagen der Lesekompetenz. In U. Schiefele, C. Artelt, W. Schneider \& P. Stanat (Hrsg.), Struktur, Entwicklung und Förderung von Lesekompetenz. Vertiefende Analysen im Rahmen von PISA 2000 (S. 101-124). Wiesbaden: VS Verlag für Sozialwissenschaften.

Mullis, V. S. I., Martin, M. O., Kennedy, A. M. \& Foy, P. (2007). PIRLS 2006 International Report. IEA's Progress in International Reading Literacy Study in primary school in 40 countries. Chestnut Hill, MA: TIMSS \& PIRLS International Study Center, Boston College. 
National Institute of Child Health and Human Development (NICHD). (2000). Report of the National Reading Panel. Teaching children to read: An evidence-based assessment of the scientific research literature on reading and its implications for reading instruction (NIH Publication No. 00-4769). Washington, DC: U. S. Government Printing Office.

Notter, Ph., Meier, U., Nieuwenboom, W., Rüesch, P. \& Stoll, F. (1996). Lernziel Lesen. Lesekompetenzen von Kindern und Jugendlichen in der Schweiz. Schweizerischer Bericht über die IEA Reading Literacy Study. Aarau: Sauerländer.

Notter, Ph., Bonerad, E.-M. \& Stoll, F. (Hrsg.). (1999). Lesen - eine Selbstverständlichkeit? Schweizer "Bericht zum International Adult Literacy Survey». Chur: Rüegger.

OECD PISA. (2003). Wichtige Resultate. Bestimmungsfaktoren. OECD/PISA. Neuchâtel: Nationales Direktorium PISA/Bundesamt für Statistik.

Oelkers, J. \& Reusser, K. (2008). Qualität entwickeln, Standards sichern, mit Differenz umgehen. Eine Expertise in Auftrag von vier Ländern. Unter Mitarbeit von E. Berner, U. Halbheer \& S. Stolz. Berlin: Bundesministerium für Bildung und Forschung.

Paefgen, E. (2006). Einfuihrung in die Literaturdidaktik (2. Auflage). Stuttgart: Metzler.

Pekarek Doehler, S. (2009). Rapport final. L'organisation du discours dans l'interaction en langue première et seconde: acquisition, enseignement, évaluation. Diversité des langues et compétences linguistiqes en Suisse, NFP 56. Zugriff am 01.03.2010 unter http://www.nfp56.ch/d_portraet_resultate.cfm?Projects.Command=resultate\&pid=6

Pieper, I., Rosebrock, C., Volz, S. \& Wirthwein, H. (2004). Lesesozialisation in schriftfernen Lebenswelten. Lektüre und Mediengebrauch von HauptschülerInnen. Weinheim: Juventa.

Retelsdorf, J. \& Möller, J. (2008). Entwicklungen von Lesekompetenz und Lesemotivation: Schereneffekte in der Sekundarstufe? Zeitschrift für Entwicklungspsychologie und Pädagogische Psychologie, 40, 179-188.

Rhyn, H. (2003). Die Bedeutung der Aus- und Weiterbildung der Lehrerinnen und Lehrer im Aktionsplan «PISA 2000»-Folgemassnahmen der EDK. Beiträge zur Lehrerbildung, 21 (3), 422-429.

Rosebrock, C. (2006). Zum Verhältnis von Lesesozialisation und literarischem Lernen. Didaktik Deutsch, 6, 57-68.

Rosebrock, C. \& Nix, D. (2006). Forschungsüberblick: Leseflüssigkeit (Fluency) in der amerikanischen Leseforschung und -didaktik. Didaktik Deutsch, 12 (20), 90-112.

Rychen, D. S. \& Salganik, L. H. (2003). Key competencies for a successful life and a wellfunctioning society. Cambridge: Hogrefe \& Huber.

Schneider, H., Bertschi-Kaufmann, A., Häcki Buhofer , A., Kassis, W., Kronig, W., Beckert, C., Stalder, U. M. \& Wiesner, W. (2009). Die erfolgreiche literale Entwicklung von risikobehafteten Jugendlichen - motivationale Aspekte. Bulletin suisse de linguistique appliqué, 89, 65-97.

Schneuwly, B., Dolz, J. \& Cordeiro, G. S. (2005). À la recherche de l'objet enseigné. Une démarche multifocale. Les Dossiers des Sciences de l'Education, 14, 77-93.

Schnotz, W. \& Dutke, S. (2004). Kognitionspsychologische Grundlagen der Lesekompetenz. Mehrebenenverarbeitung anhand multipler Informationsquellen. In U. Schiefele, C. Artelt, W. Schneider \& P. Stanat (Hrsg.), Struktur, Entwicklung und Förderung von Lesekompetenz. Vertiefende Analysen im Rahmen von PISA 2000 (S. 61-99). Wiesbaden: VS Verlag für Sozialwissenschaften.

Schweizerische Koordinationsstelle für Bildungsforschung [SKBF]. (2010). Bildungsbericht Schweiz 2010. Aarau: Schweizerische Koordinationsstelle für Bildungsforschung.

Soussi, A., Petrucci, F., Ducrey, F. \& Nidegger, Ch. (2008). Pratiques déclarées d'enseignement de la lecture et performances des élèves dans le canton de Genève. Genève: Service de la recherche en éducation.

Sturm, A., Hollenstein, A. \& Hilbe, R. (2009). Literale Grundkompetenzen und Medien in der Erwachsenenbildung. Ergebnisse des Projekts Illettrismus und neue Technologien. In 
A. Bertschi-Kaufmann \& C. Rosebrock (Hrsg.), Literalität. Bildungsaufgabe und Forschungsfeld (S. 233-246). Weinheim: Juventa.

Topping, Keith J. (2006). Building reading fluency: Cognitive, behavioral, and socioemotional factors and the role of peer-mediated learning. In S. J. Samuels, A. E. Farstrup (Ed.), What research has to say about fluency instruction (p. 106-129). Newark: International Reading Association.

Trenk-Hinterberger, I., Nix, D., Rieckmann, C., Rosebrock, C. \& Gold, A. (2008). Förderung der Leseflüssigkeit bei schwachen Leser(inne)n in der sechsten Jahrgangsstufe: Erste Ergebnisse einer Interventionsstudie. In B. Hofmann \& R. Valtin (Hrsg.), Checkpoint Literacy. Tagungsband 1 zum 15. Europäischen Lesekongress 2007 in Berlin (S. 183194). Berlin: Deutsche Gesellschaft für Lesen und Schreiben.

UNESCO. (2009). Global report on adult learning and education. Hamburg: UNESCO Institute for Lifelong Learning.

Wigfield, A. \& Guthrie, J. T. (1997). Relations of children's motivation for reading to the amount and breadth of their reading. Journal of Educational Psychology, 89, 420-432.

Wolf, M. \& Katzir-Cohen, T. (2001). Reading Fluency and Its Intervention. Scientific Studies of Reading, 5 (3), 211-239.

Schlagworte: Lesekompetenz, Leseunterricht, Leseförderung, Leseleistung, Leistungsdiagnose, Literaturwerb

\section{L'enseignement de la lecture et ses orientations suite aux enquêtes PISA. Conceptions et pratiques en tension entre le développement d'une compétence quotidienne et acculturation}

Résumé

A la suite de PISA, la conception de la lecture comme un ensemble de compétences partielles et mesurables est de plus en plus répandue. Cette conception est fondée sur un modèle théorique cognitif des processus de lecture qui considère lire comme une capacité d'action sociale rattachée à des situations de la vie quotidienne.

Si cette conception est convaincante dans la perspective d'une mesure comparative de la performance, elle n'en est pas moins problématique pour l'enseignement actuel de la lecture. D'une part, elle occulte les dimensions émotionnelles et motivationnelles importantes dans l'acquisition de la lecture; d'autre part, elle est en opposition avec des traditions de formation qui privilégient la lecture littéraire.

La recherche dans le domaine de la lecture montre clairement que l'enseignement de la lecture au secondaire I doit être organisé en combinant l'exercice de la lecture, le développement de la lecture et la lecture littéraire, des données fiables attestant l'efficacité de ces trois composantes dans les pratiques scolaires. 
Mots clés: Compétence de lecture, enseignement de la lecture, qualité de l'enseignement, motivation à lire, acquisition de la littérature

\section{La formazione alla lettura e il suo sviluppo dopo gli studi PISA. Concezioni e pratiche tra l'orientamento alle competenze quotidiane e I'inculturazione}

Riassunto

Dopo gli studi PISAE possibile osservare la crescita di una rappresentazione della competenza di lettura che la vede scomponibile in sotto-competenze parziali e misurabili. Questa rappresentazione si basa su un modello teorico cognitivo del processo di lettura, nel quale la lettura viene considerata come una capacità di azione sociale collegata a specifiche situazioni quotidiane.

Questa rappresentazione può essere funzionale soprattutto da una prospettiva comparativa delle competenze, ma può risultare problematica per l'insegnamento della lettura. Da un lato infatti trascura le importanti dimensioni emozionali e motivazioni della lettura, e dall'altra, si trova confrontata con traduzioni di formazione che privilegiano la lettura letteraria.

La ricerca sulla lettura fornisce numerosi spunti per sostenere che l'insegnamento della lettura nella scuola media deve essere costruita dall'intreccio di allenamento alla lettura, promozione della lettura e lettura letteraria. La pratica didattica deve fondarsi su dati validi relativi all'efficacia di questi tre elementi.

Parole chiave: Competenza di lettura, insegnamento della lettura, qualità dell'insegnamento, motivazione alla lettura, acquisizione delle letteratura

\section{Reading instruction and its development according to the PISA study: Conceptions and practices within the strained relationship between competency orientation and enculturation}

\footnotetext{
Abstract

In the wake of PISA, a growing understanding of reading competence that breaks reading down into measurable partial competencies can be observed. This understanding is based on a cognitive theoretical model of the reading process, which conceptualises reading as a social ability to act and relates it to everyday situations of application. Intelligible as this understanding might be for compar-
} 
ative performance measurement in particular, it is nonetheless problematic for contemporary reading instruction. To begin with, it eclipses emotional and motivational aspects that are crucial from the perspective of the acquisition of reading. In addition, it stands in direct opposition to educational traditions which ascribe significance to literary reading. Reading research gives numerous indications that reading instruction on Secondary I Level should be arranged as an interplay of reading training, reading promotion, and literary reading; whereby school praxis relies on reliable findings regarding the respective efficacy of each of these three elements.

Key words: Reading competence, reading instruction, quality of teaching, motivation to read, literature appropriation 